\title{
Apparent Ferromagnetism in the 'Pinwheel' Artificial Spin Ice
}

\author{
R. Macêdo, ${ }^{1, *}$ G. M. Macauley ${ }^{1}$ F. S. Nascimento, ${ }^{2}$ and R. L. Stamps ${ }^{3,1}$ \\ ${ }^{1}$ SUPA School of Physics and Astronomy, University of Glasgow, Glasgow G12 8QQ, United Kingdom \\ ${ }^{2}$ Departamento de Física, Universidade Federal de Viçosa, Viçosa 36570-900, Minas Gerais, Brazil \\ ${ }^{3}$ Department of Physics and Astronomy, University of Manitoba, Winnipeg, MB R3T 2N2, Canada
}

(Dated: July 14, 2018)

\begin{abstract}
Magnetic artificial spin ice provides examples of how competing interactions between magnetic nanoelements can lead to a range of fascinating and unusual phenomena. We examine theoretically a class of spin ice tilings, called pinwheel, for which near degeneracy of spin configuration energies can be achieved. The pinwheel tiling is a simple but crucial variant on the square ice geometry, in which each nanoelement of square ice is rotated some angle about its midpoint. Surprisingly, this rotation leads to an intriguing phase transition; and even though the spins are not parallel to one another, a ferromagnetic phase is found for rotation angles near $45^{\circ}$. Here, magnetic domains and domain walls are found when viewed in terms of net magnetisation. Moreover, the ferromagnetic behaviour of the system depends on its anisotropy which we can control by array shape and size.
\end{abstract}

\section{INTRODUCTION}

An unusual class of magnetic material systems has appeared called 'artificial spin ice' $[1,2]$. These are carefully structured magnetic materials composed of periodic arrangements of magnetic building blocks [3]. Each magnetic block has sub-micron dimensions and arrays typically contain hundreds of elements. By tiling these tiny magnets in specific geometrical arrangements, it is possible to create physical properties and functionalities not displayed by the constituent materials [1]. For instance, it has been recently demonstrated that artificial spin ice structures can display glass-like behaviour [3, 4], configurable charge ordering [5] and topological structures that can be analogous to magnetic monopoles $[6,7]$ and Dirac strings $[8,9]$.

The square ice geometry can be described by vertices formed from four adjacent islands meeting head-on, as shown in Fig. 1(a) and (c) $[3,10]$. The 'two-in, twoout' state has the lowest energy configuration for elements interacting through stray magnetic field produced by the elements [2]. The possibility of observing experimentally thermally driven domain formation was demonstrated for Kagome and square ice geometries by Morgan et. al. [11] and Qi et. al. [12]. Emergent properties arise from the interactions between islands which, according to their shape and position, lead to correlations and competitions that generate non-trivial dynamics and complex magnetic orderings [3].

We present a system in which the interaction of neighboring spin pairs is chosen such that near degeneracy is obtained for the energies of different neighbouring spin pairs. This is done by deforming the artificial square ice into a 'pinwheel' geometry by rotating each spin around its midpoint through an angle $\vartheta$ (see Fig. 1(a) and (c)). This rotation changes the interaction between elements so that ferromagnetic ordering is energetically favoured for some angles of rotation. This is a general result first predicted by Landau and Binder in the context of Ising spins [13]. However, the discrete geometry considered here has interesting consequences on ordering processes [14], which we explore in this paper. For instance, one of the possible pinwheel tillings has been recently studing by Gliga and co-wrkers who showed how dynamic chirality can emerge in these structures and how a ferromagnetic ordering is favoured [15]. We examine the effect of array geometries on stable and meta-stable configurations of mesoscopic domains and how their growth is mediated by 'wall-like' boundaries. Because of this ferromagnetic ordering, the array as a whole can posses a shape anisotropy that can compete with the shape anisotropies of individual elements. This competition can be understood in terms of the topology of the array edges.

\section{THEORETICAL CONSIDERATIONS}

We consider element geometries where each magnetic island is a ferromagnetic single domain with strong shape anisotropy such that its magnetic moment behaves as an Ising-like spin. The lattice constant, $a$, is the distance between the centres of a spin and its next-nearest neighbour $(n n n)$ as shown in Fig. 1(a) and (c). We approximate each island as a point magnetic dipole. This approximation can capture key features of square ice systems when compared to micromagnetics or experiments. In the case of the pinwheel ice, we will see that the approximation is much more severe. In the dipole-dipole approximation, the interaction between magnetic moments in the array is given by a dipole Hamiltonian of the form $[16,17]$

$$
\mathcal{H}_{\text {dip }}=D \sum_{i \neq j} s_{i} s_{j}\left[\frac{\hat{\sigma}_{i} \cdot \hat{\sigma}_{j}}{r_{i j}^{3}}-\frac{3}{r_{i j}^{5}}\left(\hat{\sigma}_{i} \cdot \vec{r}_{i j}\right)\left(\hat{\sigma}_{j} \cdot \vec{r}_{i j}\right)\right] .
$$

where $D=\mu_{0} \mu^{2} / 4 \pi$ and the distance between two spins is given by $r_{i j}^{2}=\hat{x} \cdot\left(r_{j}-r_{i}\right)^{2}+\hat{y} \cdot\left(r_{j}-r_{i}\right)^{2}$, where the vectors $\vec{r}_{i}$ and $\vec{r}_{j}$ give the $x$ and $y$ positions of spins at the $i$-th and $j$-th site $[18,19]$.

The spin at the $i$-th site has a magnetic moment $\mathbf{S}_{\mathbf{i}}=$ $\mu s_{i} \hat{\sigma}_{i}$ where $s_{i}= \pm 1 . \mu_{i}$ is the total moment and the unit 

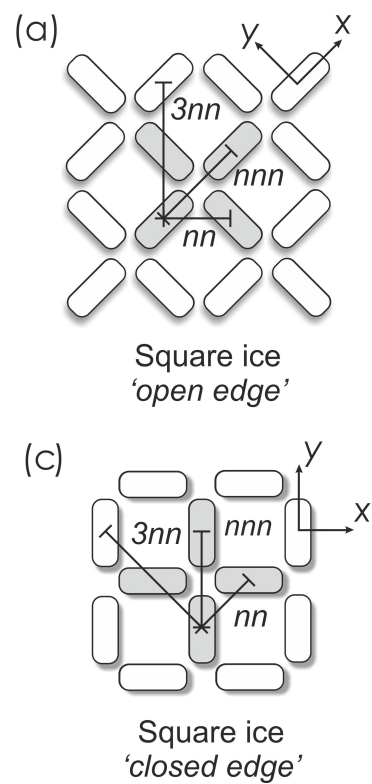

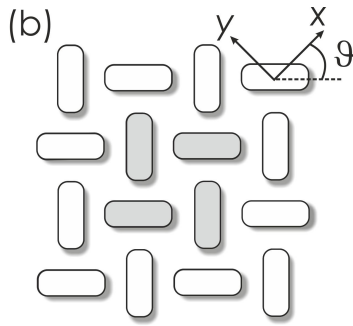

Pinwheel ice 'lucky-knot edge'

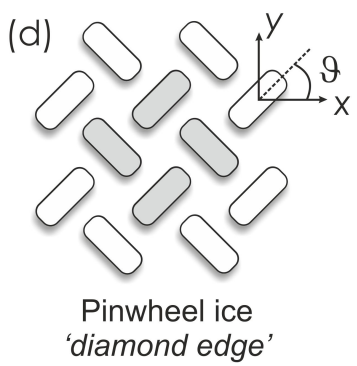

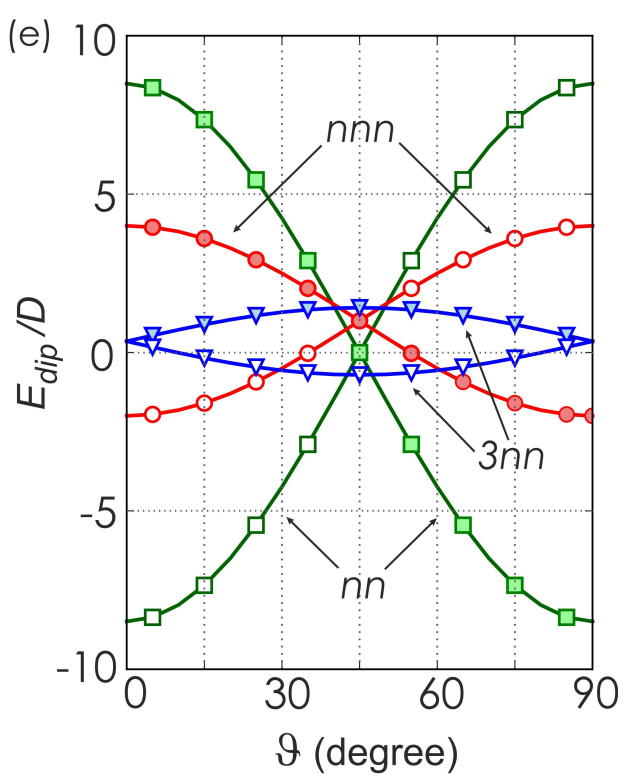

FIG. 1. Square ice lattice arrangements with (a) 'open edges' and (c) 'closed edges'. Respective arrangements at $\vartheta=45^{\circ}$ with (b) 'lucky-knot edges' and (d) 'diamond edges'. (e) Dipolar energy for $n n$, $n n n$ and $3 n n$ spin pairs as a function of $\vartheta$. Open and closed symbols are for favourably and unfavourably arranged spin pairs respectively.

vector $\hat{\sigma}_{i}$ represents its orientation specified in terms of $\vartheta$. Using the spin system depicted in Fig. 1(c), in the global frame of reference, $\hat{\sigma}_{i}=(\cos \vartheta,-\sin \vartheta)$ for the horizontal spins and $\hat{\sigma}_{i}=(\sin \vartheta, \cos \vartheta)$ for the vertical spins. We call the structure with rotated spins as 'pinwheel' and consider two possible tillings for finite arrays defined by the orientation of array edge relative to the lattice: The 'lucky-knot edge', depicted in Fig. 1(b), and the 'diamond edge', shown in Fig. 1(c).

The resulting dipolar energies for nearest neighbour $(n n)$, next-nearest-neighbour (nnn) and third nearest neighbour $(3 n n)$ spin pairs as a function of $\vartheta$ are presented in Fig. 1(e). The open and closed symbols represent the favorable and unfavorable spin pair alignments respectively. For $n n$ pairs, the interaction is maximal at $\vartheta=0^{\circ}$ but goes to zero at $\vartheta=45^{\circ}$. Thus, square spin ice $\left(\vartheta=0^{\circ}\right)$ is a system dominated primarily by nearest neighbour interactions [20] whereas pinwheel $\left(\vartheta=45^{\circ}\right)$ is a system dominated by interactions between more distant neighbours. A similar behaviour is observed for $n n n$ pairs: namely, their contribution to the total energy decreases as rotation angle increases towards $45^{\circ}$. By contrast, the dipole energy between $3 n n$ spin pairs increases with rotation angle, until it peaks at $\vartheta=45^{\circ}$. Indeed, at $\vartheta=45^{\circ}$, the strongest pair-wise interaction of any type is between $3 n n$ and all other interactions are slightly smaller. In general all interactions in pinwheel ice are of comparable magnitude out to neighbour pairs within a radius of approximately $4 a$. Therefore, throughout this work the full dipole sum with all possible neighbours is performed when evaluating Eq. 1.

\section{THERMAL PROCESSES}

In order to obtain thermodynamic averages for the Hamiltonian given in Eq. 1, we use a single-spin flip Monte Carlo approach. A single Monte Carlo step (MCS) is $L_{x} \times L_{y}$ single-spin flips these being the $x$ and $y$ lengths of the arrays (in terms of $a$ ) respectively. We have considered array sizes varying from 625 to $10^{4}$ spins. In general, $10^{4} \mathrm{MCS}$ were sufficient to reach equilibrium. For the results presented in this paper, we have used $10^{5}$ MCS to obtain the thermodynamic averages. In such cases the fluctuations in energy were never greater than $5 \%$.

For comparison we note thermal properties of a spin ice system when periodic boundaries are imposed. In Fig. 2(a), the specific heat as a function of temperature is given for various values of $\vartheta$. The specific heat, $c=\left(\left\langle E^{2}\right\rangle-\langle E\rangle^{2}\right) / k_{B} T$ where $E$ is the total energy of the system, exhibits a peak for all angles shown. If we look at the case of a square ice lattice $\left(\vartheta=0^{\circ}\right)$ a peak in the specific heat is seen at approximately $7.2 D / k_{B}$. The position of this peak is consistent with that obtained previously by Silva et. al. [16]. The ground state configuration for this case is twofold degenerate and obeys the ice rule.

Since the magnetic nano-islands can be modelled as Ising spins, it is expected that the phase transition from a disordered state to an ordered state is consistent with the well-known behaviours of Ising model transitions, such as that shown by Binder and Landau [13]. So far, we have investigated this transition by looking at the behaviour of the aforementioned specific heat $c$. This quantity is of particular interest in this work as it can be applied to all angles of rotation in the same way. However, another physical quantity, the magnetic order parameter $M$, is 
also extremely useful when characterising a novel system.
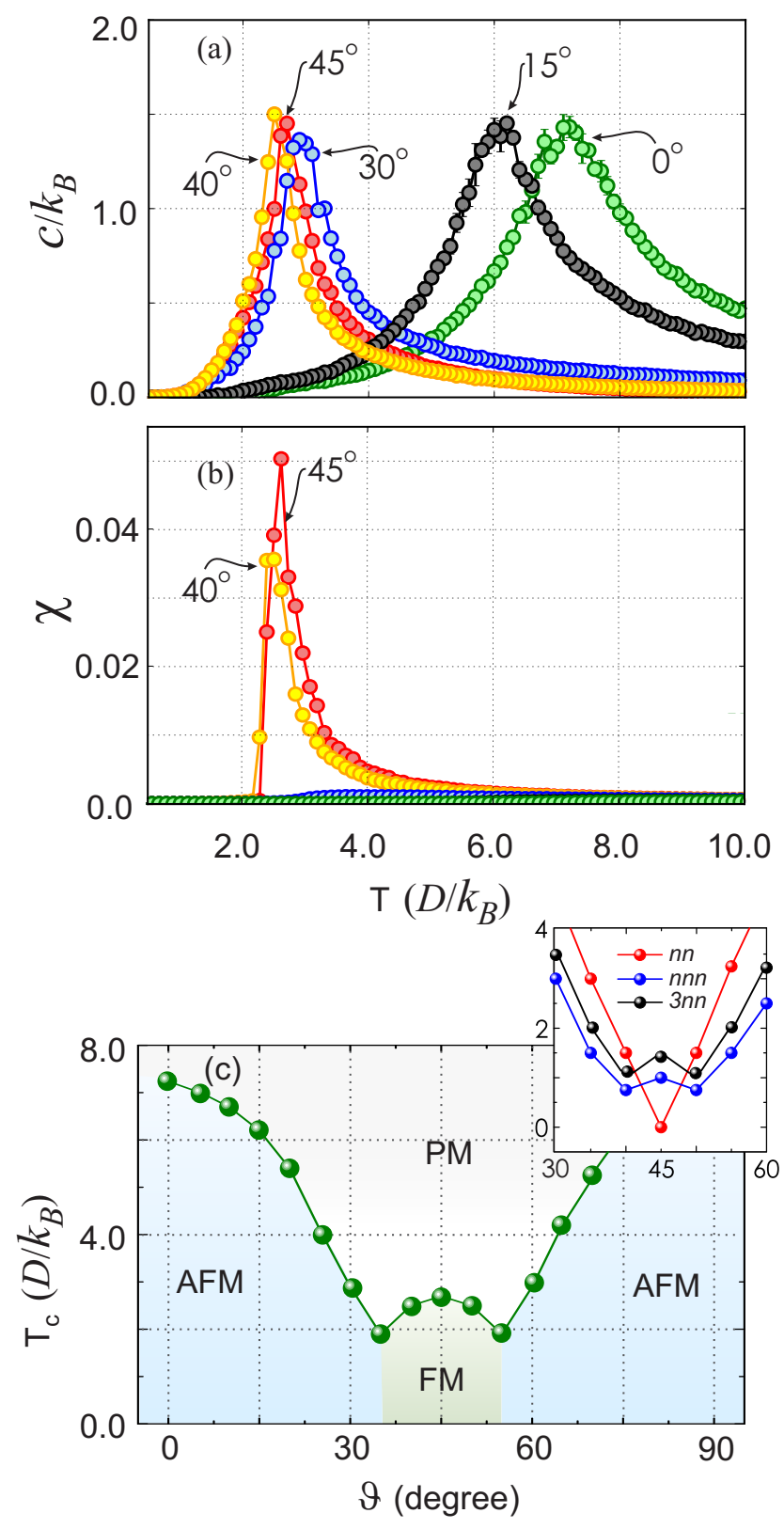

FIG. 2. (a) Heat capacities $c$ for different values of $\vartheta$ and (b) Magnetic Susceptibilities $\chi$ for various angles of rotation and as a function of temperature T. Note that only $\vartheta=45^{\circ}$ and $\vartheta=40^{\circ}$ are highlighted in part (b) as arrays at smaller angles do not have a net moment. In (c) $T_{c}$ as a function of $\vartheta$ showing a phase transition from AFM to FM is given. The inset in (c) shows the effect of considering the dipole sum only up to $n n, n n n$ and $3 n n$ when calculating $\mathrm{T}_{C}$.

For the case of classic artificial square ice geometries, this magnetic order parameter has been defined in a variety of ways. For instance, Xie et. al. has defined M as an exponential position [18] while Wysin et al defined masks for each spin vertex [21]. so that in both cases $M$ would always add up to unity at the equilibrium. There are numerous ways in which to define a susceptibility, $\chi$. We have chosen to investigate the magnetisation of a single sub-lattice in calculating

$$
\chi M(T)=\frac{\left(\left\langle M^{2}\right\rangle+\langle M\rangle^{2}\right)}{N k_{B} T}
$$

The critical temperature of $T(\vartheta)$ agrees well with that obtained from the heat capacities for those angles which possess ferromagnetic ground states. For angles outwith this ferromagnetic region, the magnetisation within each sub-lattice vanishes, and thus $\chi$ also vanishes as shown in Fig 2(b).

As the spins are rotated about their midpoint (each by the same angle, and in the same sense), the value of $T_{C}$ moves towards lower values in a non-monotonic manner in the range between $0^{\circ}$ to $45^{\circ}$. A phase diagram summarising the behaviour of $T_{C}$ as function of rotation angle is shown in Fig. 2(c). In the present case, the system transitions from a two-in, two-out ice rule ground state at $\vartheta=0^{\circ}$ to a state in which sublatices have moments aligned parallel to each other at $\vartheta=45^{\circ}$. This resembles the classical zero-field phase transition diagrams shown by Landau and Binder [13] in systems where interaction strength between neighbours decreases. An example of the resulting ferromagnetic single domain arrangement of spins at $\vartheta=45^{\circ}$ is shown in Fig. 3(b) and (f). The corresponding arrangements of the element moments are shown in Fig. 3(a) and (e). Since pinwheel elements do not meet head-on, we call the square ice vertex equivalent a pinwheel 'unit'.

There are two additional features to note from Fig. 2(c): the first is that the ferromagnetic region is quite narrow; and the second is that $T_{C}$ is never zero. The first feature is seen because the range of angles for which the system shows ferromagnetic behaviour (approximately $45^{\circ} \pm^{\circ} 8$ ) corresponds to the range for which the $n n$ interaction ceases to be the strongest relative to the other neighbour interactions (see Fig. 1(e)). The second feature is mainly due to the contributions from beyond nearest neighbours as shown in inset in Fig. 2(c). For the $\vartheta=45^{\circ}$ pinwheel, the nearest neighbour interaction is suppressed because the two moments are perpendicular to each other and the interaction cancels by symmetry in the dipole approximation. Thus, $T_{C}$ vanishes at $\vartheta=45^{\circ}$ if only $n n$ interactions are considered but is always greater than zero when interactions between more distant neighbouring spin pairs are included.

\section{EDGE EFFECTS}

We now focus our attention on the $\vartheta=45^{\circ}$ pinwheel geometry. The diamond-type array edge has the magnetisation of a domain pointing along the four cardinal points, while lucky-knot arrays edges have unit spin domains pointing along one of the four corners of the array. In the thermodynamic limit, infinite arrays uniformly 

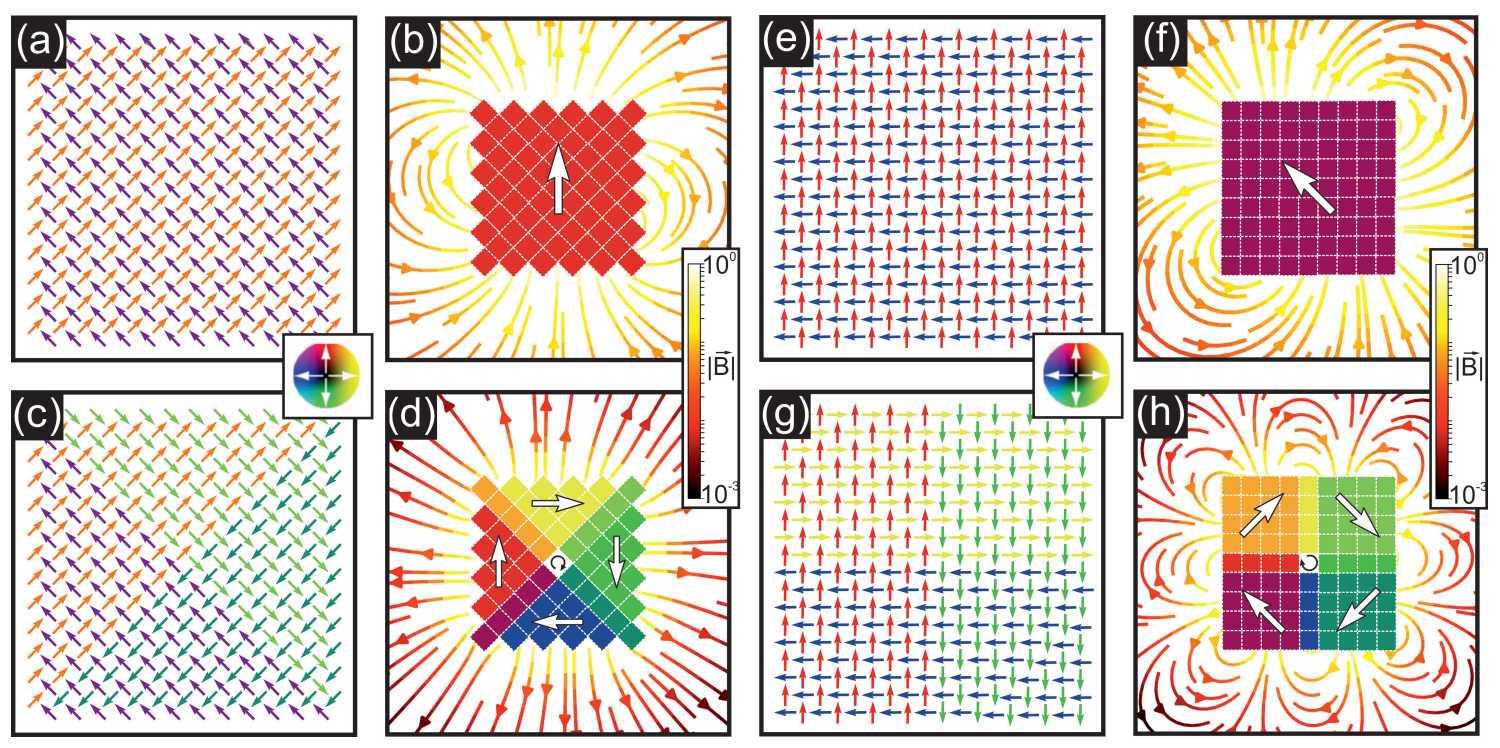

FIG. 3. Ground state configuration of (a) diamond and (e) lucky-knot pinwheel arrays with periodic boundary conditions. Parts (b) and (f) show their respective net magnetisation and stray field. Effect of (c) diamond and (g) lucky-knot edges on a finite size array. (d) and (h) shows their respective stray field.

magnetised in any of these configurations are degenerate in energy. Examples for the uniform ferromagnetic configuration are shown for reference in Fig. 3(a) and (e), for periodic boundary conditions. The effects of ' $d i$ amond' and 'lucky-knot' edges for square-shaped arrays are shown in Fig. 3(c)-(g). In both cases, a structure similar to that of a closure domain is energetically preferable over that of a single domain once the array is of sufficient size. For both edges, the critical size is at least three pinwheel units which is enough to form flux closure in the magnetisation and minimise the total energy, when compared with a uniformly magnetised array.

For the case of 'diamond' edges, a flux closure structure is preferred. This behaviour is similar to that observed in soft ferromagnetic media with cubic anisotropy $[24,25]$ as it reduces surface charges, and minimises the stray field. For comparison, we show the intensity and fieldlines of the stray field for both single domain and flux closure structures in Fig. 3(b) and (d) respectively. These plots indicate a clear reduction in the maximum intensity of the stray field. Similarly, the energy of the flux closure structure is lower than that of the single domain state for these sufficiently large arrays. The intensity of the stray field is normalised to that of the uniformly magnetised array shown in Fig. 3(b). In this case, when compared to Fig. 3(b), stronger field lines are seen and the intensity of the stray field for a closure structure decreases almost immediately beyond the array edges.

For the case of a 'lucky-knot' edges shown in Fig. 3(g), four square domains can combine to form a different flux closure structure. Unlike the 'diamond' edge case - and due to the geometry of the nano-islands - the net magnetization cannot be arranged so as to totally minimise the stray field. Even though there is not the same minimiza- tion of uncompensated charges as for the diamond edges, this state is still energetically favourable over the single domain state. We compare the equivalent stray field for both systems in Fig. 3(f) and (h). Note that the intensity decreases in a way similar to that of the diamond edge array. Because of how to the magnetisation is geometrically constrained, there are uncompensated charges and the stray field forms small loops across the array edges.

\section{ASPECT RATIO AND SIZE EFFECTS}

For pinwheel ice, the importance of the stray field on domain formation of the unit spin depends on array edge type. However, the array shape and size are also important. The global shape anisotropy of rectangular arrays, for instance, can be expected to act as a uniaxial anisotropy in analogy with continuous ferromagnetic nanobars. Thus, both array edges and aspect ratio play an important role in structure of the magnetic domains observed.

In the case of a rectangular pinwheel lattice with diamond edges, the emergent patterns resemble those seen in permalloy nanobars [22]. Fig. 4(a) shows a structure of two vortices reminiscent of diamond walls, while Fig. 4(b) describes an elongated Landau flux closure pattern. Both panels are for arrays of the same size, thermalised under the same conditions, but taken from Monte Carlo repetitions starting with different initial states. Comparing energies, we find that both configurations are nearly degenerate. Their energies differ by approximately $1.25 \%$. The closure structures form through the creation of 'mesoscopic' domain walls within the arrays i.e. domain walls whose internal structure is composed of 

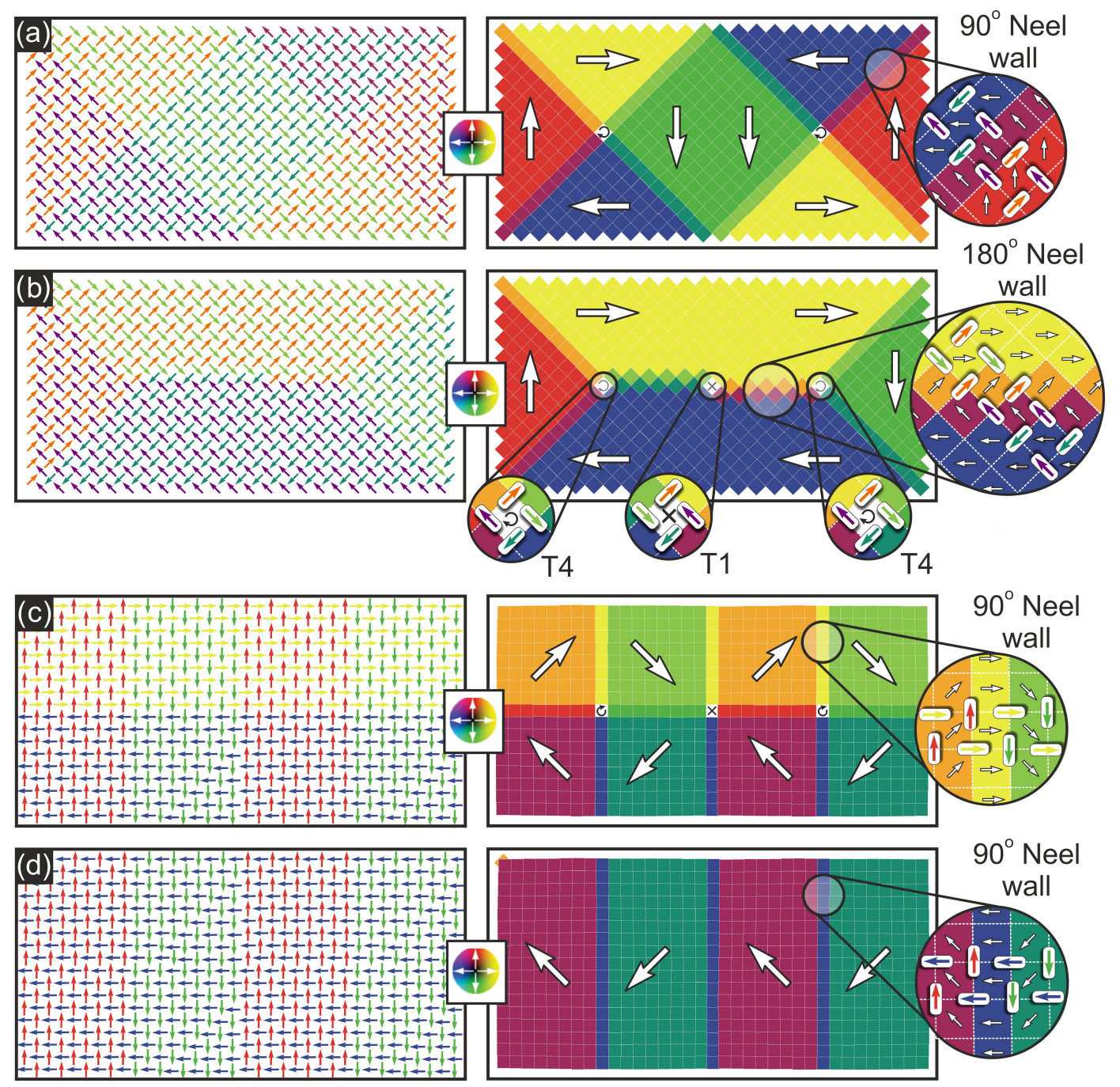

FIG. 4. To the left of (a)-(b) and (c)-(d) nearly-degenerate configurations for different thermal annealing are shown for diamond and lucky-knot edges respectively. To the right their respective unit net magnetisation orientation is given. The inset in (a) shows the formation of $90^{\circ}$ Néel walls and in (b) show, from left to right, a pinwheel unit with circular magnetisation, no net magnetisation and the formation of $180^{\circ}$ Néel walls. The insets in (c)-(d) show the formation of only $90^{\circ}$ Néel walls.

unit spins. In the first case, two such wall structures are given in the insets of Fig. 4(a) and (b). These resemble classical Néel walls in which the net moment of the unit reorients direction in the plane of the array. The cost in creating such a $90^{\circ}$ wall unit is higher than that of creating a simple ferromagnetic unit of aligned net moments. However, this cost is compensated by the reduction in global array stray field.

When a Landau flux closure pattern is formed, another type of wall is observed. These are analogous to $180^{\circ}$ Néel walls in continuous media. These walls are made of two pinwheel units and therefore the energy-per-spin is higher than a single ferromagnetic unit and a $90^{\circ}$ Néel wall unit. However, the total energy of the system is still smaller than a single magnetised domain due to the minimisation of uncompensated charges.

We note that the number of vortices in a diamond structure is related to the aspect ratio. Here, we have chosen an aspect ratio of 2:1 where no more than two vortices are formed - forming more than two vortices does not bring about a significant enough reduction in the energy associated with stray field compared with the cost in forming the walls themselves. Different aspect ratios (given in supplementary [27]) have analogous structure or more complex combinations of the structures shown in Fig. 4(a)-(b) depending on dimension.

We have mentioned previously that the effect of increasing the array size in a square shaped array of pinwheels with lucky-knot edges is to facilitate formation of smaller domains. This is shown for a single closed flux vortex structure of a small array in Fig. 5(a) becomes four closed flux vortex structures in an array with four times as many elements. However, even though the four vortex structure is a possible configuration, it forms only 
when the spin system is sufficiently large. The threshold array size depends on balancing the magnetostatic energies arising from uncompensated charges at the edges with the magnetostatic costs of internal charge distributions associated with the mesoscopic domain boundary wall structures.

(a)

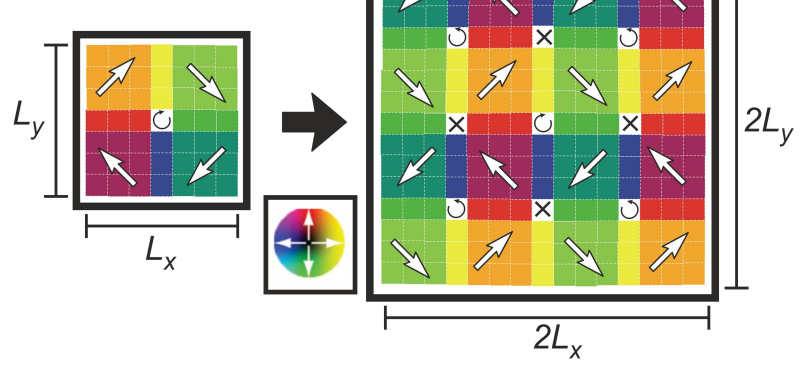

(b)
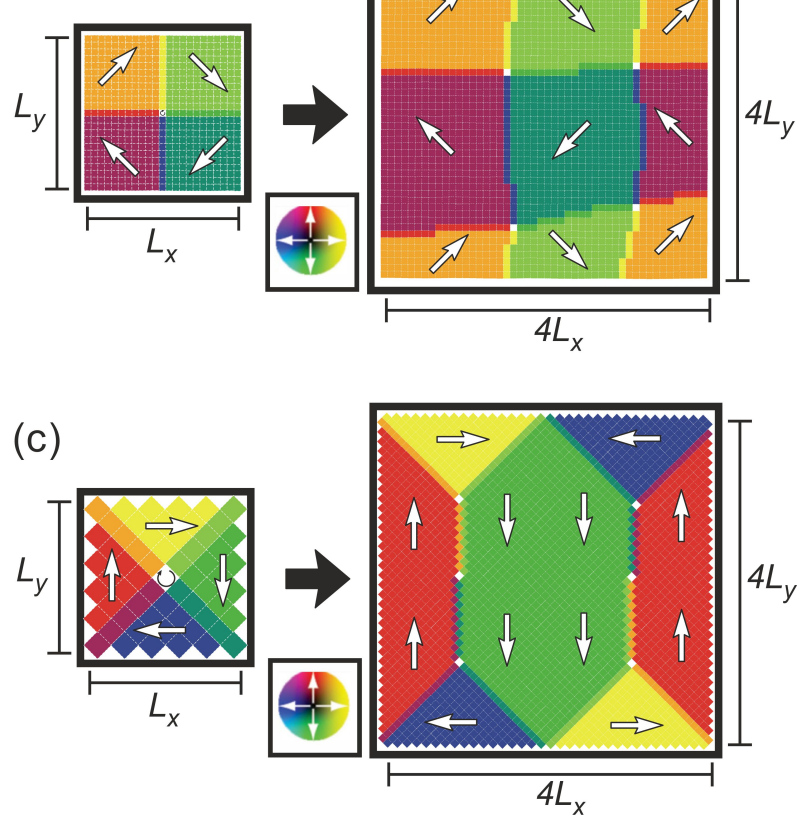

FIG. 5. Effect of size on square shaped arrays: (a) schematics of the domains breakdown as the array size is doubled and (b) simulation of doubling the array size from $25 \times 25$ to $50 \times 50$ (in units of vertices) for arrays with lucky-knot edges. The magnetisation configuration in a large array with diamond edges is shown in (c).

In large spin systems, such as the one depicted in Fig. 5(b), there are several possible arrangements which also minimize the total energy. This leads to long lived metastable state formation because once the system gets stuck in such a meta-stable state it is unlikely that a large number of spin will flip again in order to form the arrangement shown in Fig. 5(a). In the language of continuous film ferromagnets, the pinning strengths are large for the artificial spin ice. Pinning for the artificial spin ice is intrinsic in that it originates from the coerciv- ity of individual elements. This can be large, but is also something that can be controlled in the design of arrays.

In the case of square shaped arrays of pinwheels with diamond edges, the single flux closure magnetisation vortex is always favorable. This is because the the magnetisation structure, differently from that seen in lucky-knot edges, always minimizes the stray field. However, as the size of the array increases, a combination of multiple Landau patterns can also be achieved as seen in Fig. 5(c). This is also consistent with the bahaviour of continuous ferromagnet films. As observed in measurements done by Cherifi et. al in Co films, the probability of finding double Landau patterns in square-shaped films increases with the lateral dimensions of the film [23].

\section{DISCUSSION AND CONCLUSIONS}

In this work we have shown how multiple possible patterns can exist with nearly the same energy as the unit types that compose walls, vortex (and anti-vortex) centres, and domains all have similar energy. These degeneracies can be understood by examining the dipolar energy of the different pinwheel unit types shown in Fig. 6. At $\vartheta=0^{\circ}$, the energies are well separated with the energy of a Type 1 much lower than all others. As the angle increases, all these energies approach zero and become nearly degenerate $[26,27]$. At a narrow range of angles around $\vartheta=45^{\circ}$ the energy of a Type 2 is now lower than all the others. However, the domain walls are formed of Type $3 \mathrm{~s}$ and the centre of vortices is composed of a single Type 4 . This is a consequence of the preference of the system for a magnetisation configuration which minimises the stray field and total energy.

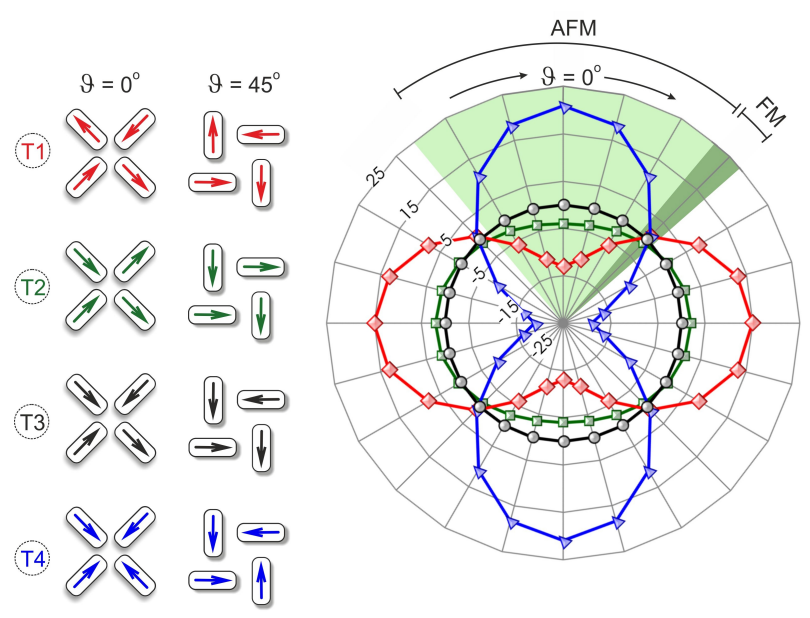

FIG. 6. Pinwheel unit types and respective dipolar energies as a function of the rotation angle $\vartheta$.

In Figs. 4(c)-(d), we show the effect of lucky-knot edges on rectangular arrays comparable in size to those of diamond. The arrangement of Fig 4(c) shows a combination of two of the vortices shown in Fig. 3(b). In order to ar- 
range the magnetisation in this way, it is necessary to form an anti-vortex in the middle of the array. For the lucky-knot array edge, only $90^{\circ}$ Néel walls are seen. This is the same as that observed in the diamond-edge case (though rotated through by $45^{\circ}$ ). These unusual vortices are formed by breaking one large ferromagnetic domain into small square ferromagnetic domains with circulating direction of net magnetisation.

In contrast to diamond-edge arrays, the lucky-knot edges do not promote the formation of $180^{\circ}$ Néel walls. Instead, the system often prefers to form a state with a net moment parallel to the long-axis of the array, but which is broken up into several domains.

Our results show that the pinwheel spin ice can behave as a 'macro-ferromagnet', despite its non-collinear spins, with a tendency to form domains and domain walls analogous to those in continuous ferromagnetic materials [23-25]. In the cases we studied, only $90^{\circ}$ and $180^{\circ}$ Néel walls have been observed in the ground state configurations. However, experimental work on pinwheel arrays shows that a variety of unusual charge ordered domain walls is possible in field driven magnetization processes [14]. Similar wall structures have also been observed in our calculations during the thermal annealing. However, the magnetization configurations induced by such walls are not stable against thermal fluctuations.

The unit geometry defines two easy and two hard axes which can compete with the shape anisotropy. This is what drives the different domain structures observed for lucky-knot and diamond edges. Moreover, the variety of stable and metastable configurations affects avalanche dynamics during domain growth and creates sensitivity to array edges.

Finally, interactions at the critical angle at which the system changes from antiferromagnetic to ferromagnetic prevent $\mathrm{T}_{c}$ from going to zero. Such behaviour is not seen in the system investigated by Binder and Landau. We believe this indicates potential for frustration between phases and the possibility of suppression of magnetic order around these angles.
* Rair.Macedo@glasgow.ac.uk

[1] C. Nisoli, R. Moessner and P. Schiffer, Rev. Mod. Phys. 85, 1473 (2013).

[2] L. J. Heyderman, and R. L. Stamps, J. Phys.: Condens. Matter 25363201 (2013).

[3] R. F. Wang, C. Nisoli, R. S. Freitas, J. Li, W. McConville, B. J. Cooley, M. S. Lund, N. Samarth, C. Leighton, V. H. Crespi and P. Schiffer, Nature 439, 303-306 (2006).

[4] A. P. Ramirez, A. Hayashi, R. J. Cava, R. Siddharthan and B. S. Shastry, Nature 399, 333-335 (1999).

[5] Y.-L. Wang, Z.-L. Xiao, A. Snezhko, J. Xu, L. E. Ocola, R. Divan, J. E. Pearson, G. W. Crabtree, W.-K. Kwok, Science 352, 962-966 (2016)).

[6] C. Castelnovo, R. Moessner, and S. L. Sondhi, Nature 451, 42-45 (2008).

[7] H. Kadowaki, N. Doi, Y. Aoki, Y. Tabata, T. J. Sato, J. W. Lynn, K. Matsuhira, and Z. Hiroi, J. Phys. Soc. Jpn 78, 103706 (2009).

[8] L. D. C. Jaubert and P. C. W. Holdsworth, Nature Phys. 5, 258-261 (2009).

[9] D. J. P. Morris, D. A. Tennant, S. A. Grigera, B. Klemke, C. Castelnovo, R. Moessner, C. Czternasty, M. Meissner, K. C. Rule, J.-U. Hoffmann, K. Kiefer, S. Gerischer, D. Slobinsky and R. S. Perry, Science 326, 411-414 (2009).

[10] G. Moller and R. Moessner, Phys.Rev. Lett. 96, 237202 (2006).

[11] J. P. Morgan, A. Stein, S. Langridge and C. H. Marrows, Nat. Phys. 7, 75-79 (2011).

[12] Y. Qi, T. Brintlinger, and J. Cumings, Phys. Rev. B 77, 094418 (2008).

[13] D. P. Landau and K. Binder, Phys. Rev. B 31, 5946 (1985).

[14] Y. Li, G. W. Paterson, F. S. Nascimento, G. M. Macauley, C. Ferguson, S. A. Morley, D. A. MacLaren, R. Macêdo, C. H. Marrows, S. McVitie and R. L. Stamps, Ferromagnetism and domain-wall topologies in artificial 'pinwheel' spin ice Private Communication (2018).

[15] S. Gliga et al., Nat. Mat. 16, 1106-1111 (2017).
[16] R. C. Silva, F. S. Nascimento, L. A. S. Mol, W. A. MouraMelo and A. R. Pereira, New Journal of Physics 14, 015008 (2012).

[17] J. M. Porro,S. Morley, D. A. Venero, R. Macêdo, M. C. Rosamond, E. H. Linfield, R. L. Stamps, C. H. Marrows and S. Langridge, arXiv:1710.03018 (2017).

[18] Y.-L. Xie, Z.-Z. Du, Z.-B. Yan and J.-M. Liu, Sci. Rep. 5, 15875 (2015).

[19] G. M. Wysin, W. A. Moura-Melo, L. A. S. Mol and A. R. Pereira J. Phys.: Condens. Matter 24, 296001 (2012).

[20] C. Nisoli, J. Li, X. Ke, D. Garand, P. Schiffer and V. H. Crespi, Phys. Rev. Lett. 105, 047205 (2010).

[21] G. M. Wysin, A. R. Pereira, W. A. Moura-Melo and C. I. L. de Araujo, J. Phys.: Condens. Matter 27076004 (2015).

[22] S. McVitie and J. N. Chapman, MRS Bulletin, 20 (10), 55-58 (1995).

[23] S. Cheriff, R. Hertel, J. Kirschner, H. Wang, R. Belkhou, A. Locateli, S. Heun, A. Pavlovska and E. Bauer, J. App. Phys. 98, 043901 (2005).

[24] L. D. Landau and E. Lifshitz, Phys. Z. Sowejetunion 8, 153 (1935).

[25] C. Kittel, Phys.Rev. 70, 965 (1946).

[26] M. J. Morrison, T. R. Nelson, C. Nisoli, New J. Phys. 15 (4), 045009 (2014).

[27] See Supplemental Material at [URL will be inserted by publisher] for extra details on the energy levels as a function $\vartheta$ for various theoretical methods as well as details on domain wall formation in larger arrays

The authors have benefited from useful discussions with R. L. Silva, J. N. Chapman and S. McVitie. Thanks are also due to $\mathrm{Y}$. Li and co-workers for privately sharing their unpublished experimental data on domain wall formation in pinwheel artificial ice. F.S.N. has benefited 
from discussion with A. R. Pereira on the methods as well as discussions of the data. This work was supported by the Engineering and Physical Sciences Research Council (EPSRC grant number EP./L002922/1) and the Univer- sity of Glasgow. The work of G.M.M. was also supported by the Carnegie Trust for the Universities of Scotland. R.L.S. also received partial support from the University of Manitoba. 